\title{
ARE REDSHIFTS OF SEYFERT GALAXIES COSMOLOGICAL?
}

\author{
A.K. SAPRE and P.S. PARIHAR \\ School of Studies In Physics, Pt. Ravishanker Shukla University, Raipur 492010, India
}

\begin{abstract}
We have studied the Hubble diagram for an almost complete sample of 48 Seyfert galaxies. We find that if Malmquist bias is taken into account, the correlation of $[\log (c z), V]$ pairs for Seyfert galaxies is statistically significant and that the slopes of the two regression lines are consistent with those expected if the redshifts of Seyfert galaxies are cosmological.
\end{abstract}

\section{The Hubble Diagram of Seyfert Galaxies}

The Hubble diagram, i.e., $[\log (c z), V]$ plot for Seyfert galaxies is generally considered to be not very enlightening (Weedman 1976) to show that the redshifts of Seyfert galaxies are proportional to their distances. This is mainly because such a diagram turns out to be a scatter diagram. In this paper we have studied the Hubble diagram for an almost complete sample of 48 Seyfert galaxies (Mackenty 1990) taking Malmquist bias into account. For this sample we find that the regression coefficient for the linear regression of $\log (c z)$ on $V$ is $b_{1}=0.133 \pm 0.024$ and that for the linear regression of $V$ on $\log (c z)$ is $b_{2}=2.889 \pm 0.525$. Though the correlation coefficient $r=0.62$ is statistically significant the two regression coefficients are not statistically consistent with the expected values of 0.2 and 5.0 respectively if the redshifts of Seyfert galaxies are cosmological. To minimise the effect of Malmquist bias we have considered only those Seyfert galaxies which lie to the brighter side of the best-fit line $V=5 \log (c z)-5.73$. For this sub-sample of 25 Seyfert galaxies we find that the regression coefficient for the linear regression of $\log (c z)$ on $V$ is $b_{1}=0.157 \pm 0.018$ and that for the linear regression of $V$ on $\log (c z)$ is $b_{2}=3.704 \pm 0.655$. The correlation coefficient $r=0.76$ is statistically significant at $99 \%$ confidence level. We find that the departure of the two regression coefficients from the theoretically expected slopes of 0.2 and 5.0 respectively is not statistically significant, at $\mathbf{9 5 \%}$ confidence level, implying that the redshifts of Seyfert galaxies are cosmological in nature.

\section{References}

Mackenty, J. W. 1990, Ap. J. Suppl. Series 72, 231.

Weedman, D. W. 1976, Q. J. R. Astr. Soc. 17, 227.

T. J.-L. Courvoisier and A. Blecha: Multi-Wavelength Continuum Emission of AGN, 520.

(c) 1994 IAU. Printed in the Netherlands. 